\title{
Increased mean platelet volume is associated with coronary artery ectasia
}

\author{
Serafettin Demir ${ }^{1}$, Mustafa Kemal Avsar², Zeynep Karakaya ${ }^{1}$, Murat Selcuk³ ${ }^{3}$ Ayd n Rodi Tosu³, Gulcan Abal', \\ Eda Tokuccu4, Mesut Demir ${ }^{5}$
}

${ }^{1}$ Adana State Hospital, Adana, Turkey

2Taksim German Hospital, Istanbul, Turkey

${ }^{3}$ Van Education and Research Hospital, Van, Turkey

${ }^{4}$ Adana Acıbadem Hospital, Adana, Turkey

${ }^{5}$ Cukurova University, Adana, Turkey

Postep Kardiol Inter 2013; 9, 3 (33): 241-245

DOI: $10.5114 /$ pwki.2013.37502

\begin{abstract}
Aim: The present study was designed to investigate mean platelet volume (MPV) values in coronary artery ectasia (CAE) patients in comparison with individuals with coronary artery disease and normal coronary angiograms.

Material and methods: Patients with stable angina pectoris and scheduled for angiography were included in the study. Those with isolated coronary ectasia were evaluated in the coronary angiography. Mean platelet volume was measured in 126 patients (mean age: $57.2 \pm 11.3$ years) with isolated CAE, 126 patients (mean age: $56.3 \pm 10.4$ years) with coronary artery disease (CAD) and 122 control subjects (mean age: $58.1 \pm 11.1$ years). Coronary artery ectasia was defined as lack of stenotic lesion, on visual assessment, of the coronary arteries with a luminal dilatation 1.5 -fold or more of the adjacent normal coronary segments. Mean platelet volume values were recorded in all patients. Ectasia severity was evaluated and categorized in accordance with the Markis categorization.

Results: A significant difference with respect to age, sex, hypertension, diabetes mellitus, and cigarette use between the study groups was not found $(p>0.05)$. Mean platelet volume was significantly higher in patients with CAE and CAD than in the control group $(9.8 \pm 3.8 \mathrm{fl}$ and $9.7 \pm 3.9 \mathrm{fl}$ vs. $8.8 \pm 3.3 \mathrm{fl}, p<0.001)$. There were no statistically significant differences between CAE and CAD groups. When type I and type IV subgroups were compared in patients with coronary artery ectasia, MPV was detected as statistically high in the type I subgroup $(p=0.026)$.

Conclusions: It was shown that patients with CAE and CAD have higher MPVs than subjects with normal coronary angiograms. Moreover, it was detected that in coronary artery ectasias, the increase in MPV is related to both the atherosclerotic process and severity of the disease.
\end{abstract}

Key words: coronary artery ectasia, mean platelet volume, coronary angiography.

\section{Introduction}

Coronary artery ectasia (CAE) is defined as diffuse or local dilatation of the epicardial coronary arteries without obstruction [1]. A 1.5- to 2-fold dilatation of a normal coronary artery angiogram constitutes CAE; a greater than twofold increase is considered to be a coronary aneurysm $[2,3]$. Coronary artery ectasias and aneurysms are seen in about $0.3-5.3 \%$ of all diagnostic coronary angiographies. Ectasia most frequently affects the right coronary artery, followed by the circumflex branch of the left coronary artery and the left anterior descending artery. Underlying etiological caus- es include atherosclerosis (50\%), congenital origins (20-30\%), and inflammatory and connective tissue diseases (20-30\%) $[2,3]$. The increased cardiac morbidity and mortality is caused by slow blood flow, coronary vasospasm, dissection, and thrombus formation [4-6]. Atherosclerosis is thought to be the major cause of CAE $[7,8]$; coronary artery stenosis and atheromatous ulceration are common.

Mean platelet volume (MPV), an indicator of platelet activation, has an independent effect on the pathophysiology of atherosclerosis in the presence of other risk factors. An increase in MPV can be caused by substances secreted in

\section{Corresponding author:}

Serafettin Demir, Adana State Hospital, Karatas Yolu Adana Devlet Hastanesi Yüre ir, 01270 Adana, Turkey, tel.: 905336271991 ,

e-mail: demirkardiyoloji@hotmail.com

Received: 16.05.2013, accepted: 12.06.2013. 
the development of acute myocardial infarction, unstable angina pectoris, and congestive heart failure $[9,10]$. However, the role of increased MPV in isolated coronary ectasia patients is unclear.

\section{Aim}

The aim of our study is to show an association between MPV and isolated coronary artery ectasia, coronary artery disease (CAD), and normal coronary artery angiograms.

\section{Material and methods}

This study draws on 9673 consecutive patients admitted to our hospital who had had coronary angiography performed and stable angina pectoris diagnosed. Cases of isolated coronary ectasia were identified, being defined as stenosis without a lesion, or a dilatation of the coronary artery segment $\geq 1.5$ times wider than the adjacent normal segment. The angiography results were interpreted and analyzed by two independent experienced operators. There were 126 patients with isolated CAE showing ectatic coronaries without any visible stenotic lesion. The CAD group consisted of 126 age- and gender-matched subjects who were selected in a consecutive manner from the catheterized patients during the same study period and who had $50 \%$ or more stenotic lesions in coronary angiograms. The control group consisted of 122 age- and gender-matched subjects who were selected in a consecutive manner from the catheterized patients during the same study period and who proved to have normal coronary angiograms. Patient medical histories and 12-lead electrocardiogram (EKG) were assessed. Complete blood count, urea, creatinine, serum sodium, potassium, HDL, LDL, and triglyceride levels were determined. Patients with any of the following were excluded: acute coronary syndrome, previous coronary artery bypass grafting, a history of antiplatelet or anticoagulant use or percutaneous coronary intervention, myocardial infarction, left ventricular systolic dysfunction, left ventricle hypertrophy, renal and hepatic deficiency (serum creatinine $>2.0 \mathrm{mg} / \mathrm{dl}$ and ASTALT > 2 times the normal value), hypothyroidism, hyperthyroidism, thrombocytopenia, hemolytic failure, autoimmune or neoplastic disease, recent major surgery or systemic failure, respiratory tract disease (chronic obstructive pulmonary disease, chronic bronchitis, pulmonary embolism), primary pulmonary hypertension, isolated right heart insufficiency, congenital heart disease and advanced stage valve disease. The included patients were evaluated in terms of age, sex, and risk factors. Hypertension was defined as systolic blood pressure of $140 \mathrm{~mm} \mathrm{Hg}$ or above, diastolic blood pressure of $90 \mathrm{~mm} \mathrm{Hg}$ or above, or use of antihypertensive medication. Diabetes mellitus was defined as fasting blood sugar of $126 \mathrm{mg} / \mathrm{dl}$ or above, current antidiabetic treatment, or application of a diet. Patients who were smoking 10 or more cigarettes per day at the time of diagnosis were defined as smokers.

\section{Coronary angiography}

Coronary angiography was performed by the Judkins or Sones technique without the use of nitroglycerin using 6-French right and left heart catheters. Angiograms were analyzed by two blinded interventional cardiologists without knowledge of the clinical status or laboratory measurements. Coronary diameter was measured as the maximum diameter of the ectatic segment using a computerized quantitative coronary angiographic analysis system. The definition of CAE was that used in the Coronary Artery Surgery Study [1-3], defined as above. When there was no identifiable adjacent normal segment, the mean diameter of the corresponding coronary segment in the control group served as the normal value. The severity of ectasia was evaluated and categorized according to Markis [8]. In decreasing order of severity, diffuse ectasia of two or three vessels was classified as type I, diffuse disease in one vessel and localized disease in another vessel as type II, diffuse ectasia of one vessel only as type III and localized or segmental ectasia as type IV.

\section{Biochemical measurements}

Blood samples were drawn after a fasting period of $12 \mathrm{~h}$. Glucose, creatine, and lipid profile were determined by standard methods. Mean platelet volume was measured in a blood sample collected in dipotassium EDTA tubes. An automatic blood counter (A Cell-Dyn 3500, Abbott) was used for whole blood counts.

\section{Statistical analysis}

Continuous variables are given as the median \pm SD, and categorical variables are defined as percentages. To compare continuous variables, the Student $t$-test or Mann-Whitney $U$ test was used where appropriate. Categorical variables were compared with the $\chi^{2}$ test. The data in the four groups were compared with one-way ANOVA followed by the Tukey HSD test for multiple comparisons. Statistical significance was defined as $p<0.05$. The SPSS for Windows ver. 15.0 statistical software (SPSS Inc, Chicago, IL, USA) was used for all statistical calculations.

\section{Results}

The three groups were stratified by the following characteristics: age, sex, smoking, height, weight, heart rate, systolic blood pressure, diastolic blood pressure, diabetes mellitus, and hypertension rates. No statistically significant differences existed within these subcategories $(p>0.05$, Table 1).

Mean platelet volume in the CAE and CAD groups was greater than in the NCA group (both $p<0.05$ ). There were no differences between CAD and CAE group MPV values (Table 2).

Ectasia was seen $63 \%$ of patients $(n=79)$ in one artery, $33 \%$ of them $(n=42)$ in two arteries and in $3 \%$ of them $(n=4)$ in three arteries. In one case there was ectasia in 
Table 1. Demographic characteristics of patients

\begin{tabular}{|c|c|c|c|c|c|}
\hline \multicolumn{2}{|l|}{ Variable } & CAE & $C A D$ & NCA & Value of $p$ \\
\hline \multicolumn{2}{|c|}{ Age, mean \pm SD [years] } & $57.2 \pm 11.3$ & $56.3 \pm 10.4$ & $58.1 \pm 11.1$ & 0.425 \\
\hline \multirow[t]{2}{*}{ Sex, $n(\%)$} & Female & $59(46.8)$ & $69(54.8)$ & 63 (51.6) & \multirow[t]{2}{*}{0.447} \\
\hline & Male & $67(53.2)$ & $57(45.2)$ & $59(48.4)$ & \\
\hline \multicolumn{2}{|c|}{ Smoking, $n(\%)$} & $60(47.6)$ & $63(50.0)$ & $63(51.6)$ & 0.516 \\
\hline \multicolumn{2}{|c|}{ Height, mean \pm SD } & $171.1 \pm 8.7$ & $169.5 \pm 9.2$ & $169.0 \pm 8.9$ & 0.175 \\
\hline \multicolumn{2}{|c|}{ Weight, mean \pm SD } & $85.3 \pm 15.9$ & $84.1 \pm 15.5$ & $83.8 \pm 14.7$ & 0.721 \\
\hline \multicolumn{2}{|c|}{ Heart rate, mean $\pm S D$} & $84.7 \pm 16.3$ & $84.4 \pm 16.6$ & $86.4 \pm 16.3$ & 0.593 \\
\hline \multicolumn{2}{|c|}{ SBP, mean \pm SD } & $126.8 \pm 20.5$ & $126.4 \pm 18.5$ & $127.4 \pm 18.8$ & 0.927 \\
\hline \multicolumn{2}{|c|}{$\mathrm{DBP}$, mean $\pm \mathrm{SD}$} & $80.0 \pm 13.5$ & $80.0 \pm 13.4$ & $80.5 \pm 13.8$ & 0.918 \\
\hline \multicolumn{2}{|c|}{ Hematocrit, mean \pm SD } & $40.8 \pm 4.6$ & $40.9 \pm 4.4$ & $40.3 \pm 4.9$ & 0.598 \\
\hline \multicolumn{2}{|c|}{ Urea, mean \pm SD } & $31.4 \pm 6.8$ & $32.1 \pm 6.6$ & $32.3 \pm 7.6$ & 0.570 \\
\hline \multicolumn{2}{|c|}{ Hyperlipidemia, n (\%) } & $30(23.8)$ & $33(26.1)$ & $28(25.0)$ & 0.578 \\
\hline \multicolumn{2}{|l|}{$\mathrm{DM}, n(\%)$} & $27(21.4)$ & $29(23.0)$ & $26(21.3)$ & 0.936 \\
\hline \multicolumn{2}{|l|}{$\mathrm{HT}, n(\%)$} & 39 (31.0) & $33(26.2)$ & $39(32.0)$ & 0.566 \\
\hline
\end{tabular}

$C A E$ - coronary artery ectasia, $C A D$ - coronary artery disease, NCA - normal coronary artery, SBP - systolic blood pressure, DBP - diastolic blood pressure, DM - diabetes mellitus, HT - hypertension

the left main coronary artery. The right coronary artery was the artery more affected by ectasia both alone and in combination with other arteries (54\%) $(p=0.001)$. Forty-four percent of ectasia was seen in the circumflex artery (Cx) and $40 \%$ in the left anterior descending coronary artery (Table 3). There was no difference between Markis type 1 subgroups and types II and III. When type I and type IV subgroups were compared, MPV was greater in the type I subgroup ( $p=0.026)$ (Table 4, Figure 1$)$.

\section{Discussion}

Patients with coronary artery ectasia had a greater MPV than those with normal coronary arteries but not greater than those with coronary artery disease. The increase in platelet volume parallels the increase of metabolic and enzymatic activity, including increased thromboxane A2 and B2 activity per volume and glycoprotein IIb-IIla expression [11-14]. These platelets have an increased response to ADP and decreased response to prostacyclin aggregation [11, 13]. Wide platelets are dense. Alpha granules such as platelet factor 4, P-selectin [11] and platelet-derived growth factor, which contribute chemotactic and vascular neointimal proliferation as mitogenic factors, are larger than in normal platelets [15]. These factors affect cell adhesion, aggregation and thrombosis via inflammation and endothelial function. Mean platelet volume is increased in acute myocardial infarction $[16,17]$, unstable angina pectoris $[18,19]$ and congestive heart failure [16].

The observations point to the possibility that both ectasia and stenosis are formed from the same basic pathophysiology. Typically, ectasia shows diffuse hyalinization, lipid deposition, destruction of intima and media, and regional calcification. Fibrosis, cholesterol crystals and intramural bleeding are seen. These histological changes are sim-
Table 2. Evaluation of MPV between groups

\begin{tabular}{lcccc} 
& CAE & CAD & NCA & Value of $p$ \\
\hline MPV, mean \pm SD $[\mathrm{fl}]$ & $9.8 \pm 3.8$ & $9.7 \pm 3.9$ & $8.8 \pm 3.8$ & $>0.001$
\end{tabular}

Table 3. Distribution of ectatic arteries

\begin{tabular}{lc} 
Retained coronary artery & $\begin{array}{c}N=126 \\
n(\%)\end{array}$ \\
\hline$R C A$ & $32(25.4)$ \\
\hline$C x$ & $25(19.8)$ \\
\hline$L A D$ & $22(17.5)$ \\
\hline$R C A+C x$ & $18(14.3)$ \\
\hline$R C A+L A D$ & $14(11.1)$ \\
\hline$L A D+C x$ & $10(7.9)$ \\
\hline 3 veins & $4(3.2)$ \\
\hline Left main coronary artery & $1(0.8)$
\end{tabular}

$C x$ - circumflex artery, $L A D$ - left descending coronary artery, RCA right coronary artery

Table 4. Type of coronary ectasia severity

\begin{tabular}{lc} 
Type & $n(\%)$ \\
\hline I & $26(20.6)$ \\
\hline II & $30(23.8)$ \\
\hline III & $38(30.2)$ \\
\hline IV & $32(25.4)$
\end{tabular}

Type 1 - diffuse ectasia of two or three vessels, type 2 - diffuse ectasia in one vessel and localized ectasia in another vessel, type 3 - diffuse ectasia of one vessel only, type 4-localized or segmental ectasia (group 4) 


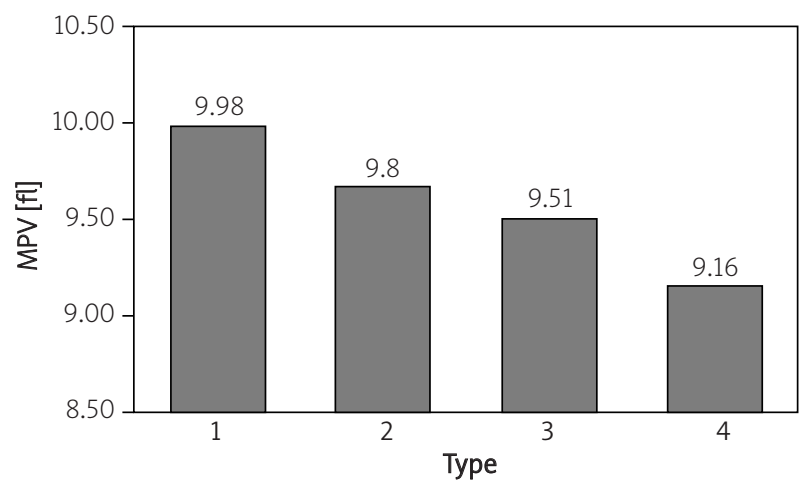

Fig. 1. Mean platelet volumes (MPV) in the subgroups of patients with $C A E$

ilar to those seen in atherosclerosis, where invasion of the media results in the destruction of musculoelastic elements and thinning of the arterial wall. Ectasia was not found in lesions where the media is unaffected [19-21].

The increase in MPV in our patients was independent of other risk factors such as diabetes, hypertension, obesity and smoking [22-26]. In the study of Sen et al., mean platelet volume was greater in 67 patients with coronary artery ectasia than in a control group. In this study MPV was greater in coronary artery ectasia patients [27]. Varol et al. evaluated mean platelet volume in coronary artery ectasia. Mean platelet volume was again greater in patients with coronary artery ectasia [28]. We also found that MPV was greater in coronary artery ectasia compared to controls. Mean platelet volume was the greatest in subgroup type-I individuals with coronary artery ectasia, although this was not found by Sen et al., who suggested that increased MPV was associated with the atherosclerotic process rather than the severity of the ectasia [27]. We feel however that MPV is related to both the atherosclerotic process and severity of the disease.

Mean platelet volume has been shown to be an independent risk factor for acute myocardial infarction [17]. Pizzulli et al. came to a similar conclusion when they compared patients with angina with those with non-cardiac chest pain [18]. Acute ischemic syndromes composed of unstable angina, acute myocardial infarction and sudden cardiac arrest are caused by thrombosis, in which thrombocyte activation is of vital importance. It can be expected that MPV is high in acute ischemia. McGill et al. argued that MPV is greater in patients with stable ischemic heart disease than in controls. In these patients, the thrombocytic adrenaline aggregation response is quicker [29]. The coexistence with coronary artery disease has led to the suggestion that CAE is a variant of coronary artery disease. MPV is greater in individuals with coronary ectasia.

\section{Conclusions}

Our data suggest that larger platelets, which are hemostatically more active, may play a specific role in development of coronary artery ectasia. Patients with larger platelets can easily be identified during routine haematological analysis and thus MPV may provide an important, simple, effortless, and cost-effective tool, which can be useful in predicting coronary artery ectasia.

\section{References}

1. Hartnell GG, Parnell BM, Pridie RB. Coronary artery ectasia: its prevalence and clinical significance in 4993 patients. Br Heart J 1985; 54: 392-395.

2. Falsetti HL, Carrol RJ. Coronary artery aneurysm. A review of the literature with a report of 11 new cases. Chest 1976; 69: 630-636.

3. Befeler B, Aranda MJ, Embi A, et al. Coronary artery aneurysms: study of the etiology, clinical course and effect on left ventricular function and prognosis. Am J Med 1977; 62: 597-607.

4. Kruger D, Stierle U, Herrmann G, et al. Exercise-induced myocardial ischemia in isolated coronary artery ectasias and aneurysms (“dilated coronopathy”). J Am Coll Cardiol 1999; 34: 1461-1470.

5. Mattern AL, Baker WP, McHale J, Lee DE. Congenital coronary aneurysms with angina pectoris and myocardial infarction treated with saphenous vein bypass graft. Am J Cardiol 1972; 30: 906-909.

6. Akyurek O, Berkalp B, Sayin T, et al. Altered coronary flow properties in diffuse coronary artery ectasia. Am Heart J 2003; 145: 66-72.

7. Swaye PS, Fisher LD, Litwin P, et al. Aneurysmal coronary artery disease. Circulation 1983; 67: 134-138.

8. Markis JE, Joffe CD, Cohn PF, et al. Clinical significance of coronary artery ectasia. Am J Cardiol 1976; 37: 217-222.

9. Huczek Z, Kochman J, Filipiak KJ, et al. Mean platelet volume on admission predicts impaired reperfusion and longterm mortality in acute myocardial infarction treated with primary percutaneous coronary intervention. J Am Coll Cardiol 2005; 46: 284-290.

10. Erne P, Wardle J, Sanders K, et al. Mean platelet volume and size distribution and their sensitivity to agonists in patients with coronary artery disease and congestive heart failure. Thromb Haemost 1988; 59: 259-263.

11. Befeler B, Aranda MJ, Embi A, et al. Coronary artery aneurysms: study of the etiology, clinical course and effect on left ventricular function and prognosis. Am J Med 1977; 62: 597-607.

12. Kruger D, Stierle U, Herrmann G, et al. Exercise-induced myocardial ischemia in isolated coronary artery ectasias and aneurysms (“dilated coronopathy"). J Am Coll Cardiol 1999; 34: 1461-1470.

13. Mattern AL, Baker WP, McHale JJ, Lee DE. Congenital coronary aneurysms with angina pectoris and myocardial infarction treated with saphenous vein bypass graft. Am J Cardiol 1972; 30: 906-909.

14. Akyurek O, Berkalp B, Sayin T, et al. Altered coronary flow properties in diffuse coronary artery ectasia. Am Heart J 2003; 145: 66-72.

15. Ferns GA, Raines EW, Sprugel KH, et al. Inhibition of neointimal smooth muscle accumulation after angioplasty by an antibody to PDGF. Science 1991; 253: 1129-1132.

16. Mathur A, Robinson MS, Cotton J, et al. Platelet reactivity in acute coronary syndromes: evidence for differences in platelet behaviour between unstable ang na and myocardial infarction. Thromb Haemost 2001; 85: 989-994.

17. Endler G, Klimesch A, Sunder-Plassmann H, et al. Mean platelet volume is an independent risk factor for myocardial infarction but not for coronary artery disease. Br J Haematol 2002; 117: 399-404.

18. Pizzulli L, Yang A, Martin JF, Lüderitz B. Changes in platelet size and count in unstable angina compared to stable angina or non-cardiac chest pain. Eur Heart J 1998; 19: 80-84.

19. Senaran H, lleri M, Altinba A, et al. Thrombopoietin and mean platelet volume in coronary artery disease. Clin Cardiol 2001; 24: 405-408. 
20. Demopoulos V, Olympios C, Fakiolas C, et al. The natural history of aneurysmal coronary artery disease. Heart 1997; 78: 136-141.

21. Swanton RH, Lea TM, Coltarte DJ, et al. Coronary artery ectasia, a variant of occlusive coronary arteriosclerosis. Br Heart J 1978; 40: 393-400.

22. Williams MJA, Stewart RAH. Coronary artery ectasia: local pathology or diffuse disease. Cathet Cardiovasc Diag 1994; 33: 116-119.

23. Papanas N, Symeonidis G, Maltezos E, et al. Mean platelet volume in patients with type 2 diabetes mellitus. Platelets 2004; 15: 475-478.

24. Nadar S, Blann AD, Lip GY. Platelet morphology and plasma indices of platelet activation in essential hypertension: effects of amlodipinebased antihypertensive therapy. Ann Med 2004; 36: 552-557.

25. Pathansali R, Smith N, Bath P. Altered megakaryocyte-platelet haemostatic axis in hypercholesterolaemia. Platelets 2001; 12: 292-297.

26. Kario K, Matsuo T, Nakao K. Cigarette smoking increases the mean platelet volume in elderly patients with risk factors for atherosclerosis. Clin Lab Haematol 1992; 14: 281-287.

27. Sen N, Tavil Y, Yazici HU, et al. Mean platelet volume in patients with coronary artery ectasia. Med Sci Monit 2007; 13: CR356-CR359.

28. Varol E, Akcay S, Ozaydin M, et al. Mean platelet volume in patients with coronary artery ectasia. Blood Coagul Fibrinolysis 2009; 20: 321-324.

29. McGill DA, Ardlie NG. Abnormal platelet reactivity in men with premature coronary heart disease. Coron Artery Dis 1994; 5: 889-900. 